\title{
Inflammation and Muscle Weakness in COPD: Considering a Renewed Role for Theophylline?
}

Running title: Theophylline for Inflammation

Stephen Allen ${ }^{1,2}$, Ahmed Khattab ${ }^{2}$, Michael Vassallo ${ }^{1.2}$, Joseph Kwan ${ }^{3}$

${ }^{1}$ The Royal Bournemouth Hospital, Dorset, UK

${ }^{2}$ Bournemouth University, Poole, UK

${ }^{3}$ The University of Hong Kong, Hong Kong SAR, China

Corresponding author: Professor Stephen Allen

Address: Department of Medicine, The Royal Bournemouth Hospital, Castle Lane East, Bournemouth, Dorset, BH7 7DW, UK

Tel: +441202303626

Fax: +441202704542

Email: Stephen.allen@rbch.nhs.uk 


\section{Abstract}

Background: The methyl-xanthine drug theophylline has been used for the treatment of asthma and chronic obstructive pulmonary disease (COPD) for several decades. Phosphodiesterase inhibition leading to smooth muscle relaxation is its main mode of action as a broncho-dilating agent, requiring blood theophylline concentrations close to the toxic range and a consequent problem with side effects. It also has anti-inflammatory effects that occur at relatively low concentrations.

Objective: To explore supporting evidence for how other properties of theophylline could be of considerable clinical utility, particularly in reducing weakness and overt sarcopenia, based on its antiinflammatory, steroid-sparing and immune-modulating properties.

Methods: PUBMED and MEDLINE were scanned using the search terms: theophylline, antiinflammatory, cytokine, inflammation, ageing, sarcopenia, frailty, chronic obstructive airways disease, pulmonary rehabilitation. High quality articles were selected, read in depth and discussed by the authors, then used as the basis for a proposition on the potential anti-inflammatory and antisarcopenic use of theophylline.

Findings: The anti-inflammatory properties of theophylline are mainly mediated by histone deacetylase induction in immune competent cells with a resulting shift toward the expression of a less inflamed phenotype, including a reduction of the release of pro-inflammatory cytokines and associated increase in anti-inflammatory cytokines. These effects occur at blood theophylline concentrations well below the range for direct broncho-dilatation.

Conclusion: There are potential therapeutic benefits of theophylline that are systemic and not confined to airways inflammation in COPD. There is scope for a beneficial effect in other chronic inflammatory states and post-acute inflammation with slow resolution. There is compelling evidence that theophylline could be used for anti-inflammatory adjunctive treatment to improve independent function, muscle strength and other outcomes in individuals recovering from acute inflammatory episodes, including but not limited to exacerbations of COPD, and as a pharmaceutical intervention in support of pulmonary rehabilitation.

Key words: theophylline, chronic obstructive pulmonary disease, systemic inflammation, immune modulation, sarcopenia, illness behaviour, pulmonary rehabilitation 


\section{Introduction}

Theophylline was first identified in the late $19^{\text {th }}$ century as a drug occurring naturally in tea and cocoa. It was synthesized a few years later and came into clinical use, initially as a diuretic, in 1902. Its other pharmacological properties gradually became apparent and it was used to relieve the symptoms of asthma from around 1922 [1]. The mechanisms of action of theophylline, and other methyl-xanthine drugs such as caffeine, theobromine and pentoxifylline, have progressively come to light. The rapid-action bronchodilator effect of theophylline is mainly due to phosphodiesterase inhibition and subsequent smooth muscle relaxation. Later, the antagonist effect of theophylline on adenosine receptors was demonstrated and is the principal reason for the positive inotropic and chronotropic cardiac effects observed, and the most likely explanation for the central nervous system stimulation and respiratory stimulant properties of that class of drugs [2]. Adenosine receptor antagonism probably also contributes to the bronchodilator effect and is also the mechanism whereby theophylline reduces nitrous oxide production by endothelial cells $[2,3]$.

Although theophylline, and its derivative for intravenous use, aminophylline, still have a role in the management of asthma and chronic obstructive pulmonary disease (COPD) [4], their use has been limited in recent decades by the emergence of more effective and less toxic beta-agonist and antimuscarinic bronchodilator drugs. The plasma concentrations of theophylline required for useful bronchodilatation (10-15 mg/L) are close to the overtly toxic range (>20 mg/L) at which a number of unpleasant and potentially dangerous side-effects occur, such as nausea, vomiting, tremor, cardiac arrhythmias, agitation, insomnia and seizures. Further, the elimination half-life of theophylline varies greatly with age, renal function, liver function, thyroid function, smoking, heart failure and pyrexia. The toxic effects are accentuated by a number of commonly used drugs such as erythromycin and ciprofloxacin, and rapid absorption leading to toxic blood levels can occur if an oral dose is taken with a fatty meal [5]. Consequently, interest in theophylline as a therapeutic agent in the context of obstructive airways disease has waned and is currently confined to certain adjunctive roles such as those defined in the British Thoracic Society guideline for COPD and asthma [4]. However, a growing understanding of the actions of theophylline as an immune-modulatory agent, particularly in the relatively non-toxic dose range $(5-10 \mathrm{mg} / \mathrm{L})$, opens a potential new role for the drug in ameliorating the deleterious effects of sustained systemic inflammation $[2,6-8]$ particularly on muscle function, cognition and mood after exacerbations of COPD and other pro-inflammatory events. That topic is the subject of this paper. 


\section{Search methods}

The electronic medical databases PUBMED and MEDLINE were scanned using the search terms: theophylline, anti-inflammatory, cytokine, inflammation, ageing, sarcopenia, frailty, chronic obstructive airways disease, pulmonary rehabilitation from 1990-2018. More than 500 articles were identified and 95 papers judged to be of good quality and relevant to the topic were selected to be read in depth and discussed by the authors. No other form of selection was applied. Some other sources were taken into consideration, mainly for background information. We have cited only the most important sources. The proposition presented in this paper emerged from discussions between the authors, supported by the discovered literature.

\section{Background - innate immune function and dysfunction}

\section{Innate immunity}

To set the context for the suggestions made later in this paper it is necessary to describe some key aspects of the innate immune system, the cells and cytokines of which are the initial line of defence against infection and other forms of tissue damage[9]. The chemistry and cell biology of innate immunity is complex and incompletely understood and is outside the scope of this paper. Empirical findings have varied but there is a sufficient amount of verified research to provide a useful understanding of a number of key processes. Taking pneumococcal infection as an example, in individuals with or without adaptive antibody immunity, pneumococcal antigens are initially presented to tissue macrophages and circulating monocytes both directly and as complexes bound to C-reactive protein (CRP), serum amyloid A (SAA) and toll-like proteins. In response, monocytes of pro-inflammatory phenotype release inflammatory cytokines of the interleukin-1 (IL-1) family, principally IL-1 $\beta$, interleukin-6 (IL-6) and tumour necrosis factor-alpha (TNF $\alpha$ ), probably within 1-3 hours as shown by in vitro studies $[10,11]$. There is some evidence that this process is delayed in older patients, based on studies in a clinical setting [12], but the clinical significance of that finding is not clear. By processes for which there is only a partial understanding those cytokines, other less extensively studied cytokines, and other bio-chemicals involved in innate immunity such as interferons and complement, stimulate a number of responses by a wide range of cell types that contribute to an effective elimination of the infection. The range of these pro-inflammatory effects also includes increased production of CRP and SAA by hepatocytes, increased endothelial permeability, reduced myoprotein synthesis and increased IL-6 release by various cells including monocytes, myocytes and adipocytes, reduced albumin synthesis, switching of neutrophils to a pro- 
inflammatory phenotype that results in further release of pro-inflammatory cytokines including TNF $\alpha$, down-regulation of autonomic cardiovascular reflexes, and context-variable effects on synthetic functions by other tissues and metabolic pathways including glucocorticoid and catecholamine production $[13,14]$. Lethargy and an urge to rest, sometimes referred to as sickness behaviour, are probably at least partly due to cytokine-mediated alterations in central nervous system function. This particular adverse consequence is of considerable clinical importance. Equally important, there is circumstantial evidence that pro-inflammatory cytokines directly predispose to delirium $[15,16]$, though the mechanism is not clear and other factors might be involved. There is a complex sequential effect on macrophages leading initially to further production of IL-1 $\beta$, TNF $\alpha$ and the anti-inflammatory cytokines interleukin-4 (IL-4) and IL-1 receptor antagonist (IL-1ra). Antigenstimulated monocytes are also self-stimulated to release TNF $\alpha$ and other cytokines by an autocrine mechanism and, through an influence on T-helper cell function, lead to rapid production and release of interferon gamma (IFY), which in turn contributes to the response to infection by a range of interactions with cells of the innate and adaptive immune systems. In the early and mature phases of this pro-inflammatory response the primary purposes of eliminating infection, minimising damage and the survival of the individual are being served. Once infection has been eliminated counterregulatory mechanisms switch the chemokine and cellular milieu of the innate immune system to an anti-inflammatory phase characterized by a rapid fall in IL-1 $\beta$, TNF $\alpha$, other pro-inflammatory cytokines and CRP, a transient rise in blood levels of anti-inflammatory cytokines such as IL-4, interleukin-10 (IL-10) and IL-1ra, and a return of immune cell phenotypes to mainly antiinflammatory and surveillance mode [16].

\section{Chronic inflammation, sarcopenia and ageing}

To provide background from another perspective it is important to consider the inflammatory state associated with chronic disease and old age as this closely approximates to the condition of many patients with COPD. Baseline biochemical markers of inflammation, such as IL-1 $\beta$, TNF $\alpha$ and CRP are often chronically raised 2- to 4- fold in the peripheral blood of older people, particularly those above the age of 80 years and, or, with multiple co-morbidities, compared with younger adults $[17,18]$. The reason for this is not entirely clear, but in some it can be explained by the presence of a clinically obvious chronic inflammatory pathology, with COPD as one of the commonest examples, and with a range of other disorders such as rheumatoid arthritis [19]. Similar levels of baseline TNF $\alpha$ and CRP are often found in people with other pathological states that are known to have a pro-inflammatory effect, such as central obesity, atherosclerosis or type 2 diabetes $[20,21]$. Several other conditions, 
associated with the depleted physiological state that characterizes frailty, have been shown to be accompanied by chronically raised pro-inflammatory markers, though the cause-and-effect relationship is less clear. These include Alzheimer's disease, chronic kidney disease, osteoarthritis and physical inactivity [22-24]. Some studies have described elevated baseline CRP and proinflammatory cytokines in elderly people with no apparent underlying chronic disease [19], thus supporting the contention that old age is in itself a pro-inflammatory condition. Patients with COPD have chronic inflammation in their airways and systemically. Further, an episode of exacerbation is characterized by a rise in blood inflammatory markers during and after the episode. This includes CRP and the pro-inflammatory cytokines mentioned above, as well as several other markers including IL-8 (strongly associated with lung inflammation), fibrinogen, leukotrienes, leptin, endothelin-1, and myeloperoxidase [25]. In its chronic state, persisting low-amplitude inflammation is linked to a number of important adverse outcomes, including increased all-cause mortality, reduced muscle strength, impaired ability to carry out essential activities of daily living and lower self-reported health status [26]. Further, it appears that chronic disease progression is at least partly a consequence of chronic inflammation and not simply a manifestation of it; there appears to be a complex interaction between cause and effect that is self-sustaining and deleterious [27]. A nonpulmonary example of this is the endothelial inflammation that occurs in atheromatous vascular disease, which can be envisaged as a destructive endo-vasculitis both resulting from and stimulating an enhanced systemic pro-inflammatory baseline state associated with enhanced endothelial nitrous oxide release [28]. This chronic slightly inflamed state, with the associated and probably consequent increase in vulnerability to an overtly frail clinical condition is also of clinical and functional importance in patients with COPD, particularly those who are likely to develop muscle weakness or are already overtly sarcopenic. Such individuals are thereby at risk of impaired locomotor function, worsening exercise intolerance and reduced respiratory muscle strength. This concept leads in the following paragraph to a consideration of the deterioration that occurs when an acute infection, or other source of inflammation, is superimposed.

\section{The deleterious effect of an extended acute pro-inflammatory state}

At this point we will consider the possible mechanisms whereby TNF $\alpha, \mathrm{IL}-1 \beta, \mathrm{IL}-6$ and other inflammatory bio-chemicals contribute to adverse clinical phenomena in COPD and similar conditions. An important example from the clinical perspective is inflammation-associated neuronal dysfunction. There is evidence that pro-inflammatory over-activity, and inadequate or incomplete anti-inflammatory resolution contribute to delirium, low mood and sickness behaviour, all of which 
are of considerable importance in the day-to-day clinical setting [29]. Autonomic dysfunction is another probable consequence, though interpretation is to some extent confounded by the immuno-regulatory role of autonomic neuroendocrine pathways. Receptors for several cytokines are present on neurons and glial cells, including TNF $\alpha$, IL-1 $1 \beta$, IL-6 and IL-1ra. Moreover, transport systems have been demonstrated that enable a number of cytokines to enter the cerebro-spinal fluid and brain interstitial fluid, including IL-1 $\beta$, TNF $\alpha$, IL-6 and IL-1ra [30-33]. In animal experiments it has been shown that measurable changes of behaviour, such as anorexia, hiding and sleepiness, are induced by artificial exposure to IL-1 $\beta$ and TNF $\alpha$ [34], which probably act via hypothalamic neurons. The role of cytokines in neuronal function is complex and appears to be both deleterious and protective depending on the metabolic and patho-physiological context [34-36]. Recent in vitro evidence suggests that the influence of cytokines on brain function is to a considerable extent mediated by receptors on various cell types in the blood-brain barrier, leading to changes in permeability and the secondary production of pro-inflammatory cytokines on the brain side of the barrier by glial cells [35]. In any case, the real-time association of elevated concentrations of IL-1 $\beta$, IL-6 and TNF $\alpha$ in peripheral blood with neuronal dysfunction during and after infection, particularly physiologically depleted and elderly individuals, is strongly suggestive that those cytokines are either directly or indirectly involved [37].

Though some of the adverse effects of IL-1 $\beta$ and TNF $\alpha$ are due to direct receptor-mediated changes in cell metabolism, there is evidence of widespread influences of a sustained pro-inflammatory state on other metabolic pathways and cell functions that are less clearly direct. These include changes in insulin sensitivity, increased catecholamine and glucocorticoid secretion, increased production of reactive oxygen species and nitrous oxide in endothelium, augmented production of prostaglandins and stimulation of interferon-gamma (IFY) release. Important indirect mechanisms include complement-mediated micro-coagulation as a consequence of a pro-inflammatory state [38]. The biological consequences of these changes are incompletely understood but some of the better described phenomena are of considerable importance in clinical practice, particularly the central and peripheral neuronal effects described above, reduced muscle protein synthesis, impaired myocyte function and increased capillary permeability, all of which can contribute to the syndrome of frailty and impaired function.

\section{The anti-inflammatory properties of theophylline}


More recently, the anti-inflammatory properties of theophylline and related drugs has been recognized. It was observed, in the context of COPD, that patients taking theophylline had more favourable outcomes in certain domains after acute exacerbations, particularly walking performance and a subjective feeling of being less exhausted [39]. Moreover, these effects appeared to be preserved at plasma theophylline concentrations $(<10 \mathrm{mg} / \mathrm{L}$ ) below the range required for useful bronchodilatation (10-15 mg/L) and therefore well below the toxic range $(>20 \mathrm{mg} / \mathrm{L})[39,40]$. Further, it was found that theophylline exhibited an anti-inflammatory effect locally on the inflamed airways of COPD patients, and systemically in clinical studies and in isolated tissue in the laboratory [41]. Other methyl-xanthine drugs have been shown to have similar properties, though most of the evidence pertains to theophylline. The mechanisms of action have not been completely resolved at the molecular level but it has been shown that theophylline reduces the production by monocytes and other immune cells of the pro-inflammatory cytokines tumour necrosis factor alpha (TNF $\alpha$ ) and interleukin-1 beta (IL-1 $\beta$ ) and increases the release of the anti-inflammatory cytokines IL-4 and interleukin-10 (IL-10) [42-44]. This has been shown to be mediated epigenetically through the induction of histone deactylase-dependent gene switching, leading to the expression of a less proinflammatory and more anti-inflammatory cell phenotype [43] (figure 1). This mechanism, and other associated gene switches that up- and down-regulate a range of cytokines, have been found to be dose-dependent but do not appear to require the high doses needed for bronchodilatation [42]. Interestingly, it has been observed in patients with COPD that the addition of theophylline to a standard drug regimen resulted in lower baseline C-reactive protein (CRP) levels and better functional scores compared to matched controls [45]. In that context, CRP can be seen as a broad indicator of the inflammatory state of the patient as its synthesis and release from hepatocytes are directly influenced by IL-1 and trough IL-6. It can be contended that the reduction of proinflammatory cytokines by theophylline is a feasible explanation for that observation. Theophylline antagonises endothelial adenosine receptors and the resulting reduction in nitrous oxide synthesis limits the rise in capillary permeability that can result from systemic inflammation [3]. It has also been shown to have a steroid sparing effect in acute exacerbations of COPD and asthma $[2,46,47]$, which is thought to be largely due to its anti-inflammatory properties, and probably to some extent to adjunctive bronchodilator action. Theophylline also reduces interleukin-6 (IL-6) levels in the peripheral blood of people with a number of chronic inflammatory disorders, including COPD. However, the role of IL-6 in inflammation is complex and varies with the physiological context. It is not simply a pro-inflammatory cytokine and is released as a myokine from skeletal muscle during exercise, as well as from immune cells in response to infection and other inflammatory stimuli. The rapid transient rise in IL-6 during exercise appears to have an overall anti-inflammatory effect, 
possibly through IL-6 mediated release of IL-10, and also through increasing insulin receptor sensitivity and other anti-sarcopenic effects on skeletal myocytes. By comparison, the high resting trough IL-6 levels found in peripheral blood in chronic and sub-acute inflammatory states and trauma are associated with a higher risk of delirium and an enhanced risk of sarcopenia and lower functional performance [48-55]. There is no reliable evidence of the effect of theophylline on postexercise or trough IL-6 levels in peripheral blood in subjects with COPD, other inflammatory states or frailty indicators.

\section{Implications for clinical practice}

In the paragraphs above we have presented the background science pertaining to the clinical consequences of prolonged or chronic low level systemic inflammation. In patients in a pre-frail or overtly frail condition, such as many with COPD, and in old age, it has been consistently observed that inflammation is causally linked with a number of common adverse effects. Those with the greatest impact on individuals and on service provision are muscle weakness and sarcopenia (limiting mobility and independent function, and increasing the risk of falls), sickness behaviour and lethargy (lowering mood and predisposing to social and functional withdrawal), reduced appetite (compromising nutrition and hydration) and delirium (greatly increasing dependency, direct care needs and the risk of injury). At present, there have been no properly structured clinical trials of the role of immune modulating drugs in the management of systemic inflammation. However, we contend that based on the existing clinical and laboratory observations discussed in this paper, theophylline is a potential candidate for targeted trials, with particular attention to the adverse outcomes mentioned in this paragraph. The following paragraph enlarges on that concept.

\section{Theophylline as an immune modulating drug in COPD and similar conditions}

Based on the evidence presented above we suggest that theophylline at target plasma levels between 5 and $10 \mathrm{mg} / \mathrm{L}$ could be deployed to down-regulate the pro-inflammatory state in COPD, particularly in the aftermath of acute exacerbations. This could lead to measurable and clinically significant improvements in function, particularly muscle strength, mobility and mood. The likely mediator of such benefits would be reduced production of TNF $\alpha$ and other pro-inflammatory components of the innate immune system, and concomitant, or consequential, up-regulation of the production of IL-10 and other anti-inflammatory bio-chemicals and immune cell phenotypes. We also contend that such an alteration in the overall balance of pro-inflammatory and anti- 
inflammatory cytokines will lead to earlier and more complete resolution of the post-infective or post-exacerbation systemic inflamed state frequently observed in patients with COPD, and other inflamed states. Any such benefit might be especially helpful in elderly pre-frail patients with depleted physiological reserves.

This proposition is of course based on certain reasonable suppositions. Firstly, that the adverse clinical outcomes described above are at least partly caused by persisting systemic inflammation and that by applying an intervention to reduce the duration and amplitude of that inflammation the outcomes will be more favourable. While the evidence for that is substantial it is not a proven cause and effect relationship. Our contention is also predicated on certain supportable assumptions regarding the mechanism of action. It is acknowledged that the effect of theophylline on inflammation chemistry is likely to be complex and to involve a range of inter-dependent cytokines, interferons, immune cells and other physiological systems that contribute to the control of innate immune responses, such as autonomic neuro-endocrine activity. It is not possible at present to posit a precise mechanism supported by linear biochemical logic in a molecule by molecule sequence. Indeed, it is probable, given the highly complex interactions of the innate immune system that the mechanistic order will vary between individuals and on different occasions depending on the type of stimulus, magnitude and rate of change of immune biochemical concentrations and the real-time status of associated physiological systems. However, we argue that it is not necessary to resolve all components of the mechanism in order to predict an overall directional change or aggregate shift towards a less inflamed phenotype. We argue that our interpretation is sufficiently consistent with the observed effects of theophylline on key inflammatory cytokines and appears partially to explain the favourable clinical responses of patients with asthma, COPD and sepsis. Further insights into the various stages of the mechanism should emerge with further focussed empirical research in the future. Of course, MXs are not unique in this regard. Several other drugs are known to have immune modulatory properties that could be similarly useful, including statins, thalidomide, 4-

aminoquinolines and beta-adrenergic receptor blockers [56-60]. These all have subtle multi-point-ofaction effects on cytokine activity but are limited by lack of clinical evidence in COPD, or side effects. Some targeted monoclonal antibodies (MCAs) are highly effective in specific pathological contexts, for example anti-TNF $\alpha$ drugs for rheumatoid disease [61], but their role as broader spectrum immune dampers is less clear. Roflumilast, a selective phosphodiesterase-4 inhibitor suppresses airway inflammation in COPD [62] and thereby reduces the systemic inflammatory burden, but probably has little direct effect on the systemic inflammation. Other drugs with anti-inflammatory properties, such as salicylates, corticosteroids and non-steroidal anti-inflammatory drugs (NSAIDs) largely act through cyclo-oxygenase [63] and phospholipase A2 [64] pathways that do not directly 
involve cytokine mechanisms and, with the possible exception of some NSAIDs [65], do not appear to have an anti-sarcopenic effect.

We also suggest that the predicted shortening of the post-infective, or post-exacerbation, inflammatory phase could lead to faster recovery from, or even avoidance of, important neuronal dysfunctions such as delirium, impaired balance, anorexia, low mood, lethargy and autonomic impairment, as well as reducing generalized oedema, myocyte dysfunction, sarcopenia, insulin resistance and other adverse metabolic states. It is reasonable to expect that such improvements in a patient's physiological milieu would augment the benefits of the structured exercise components of pulmonary rehabilitation and contribute to improved functional outcomes. Thereby, the treated patient is expected to have a lower risk of becoming overtly cachectic, sarcopenic, frail and dependent. In the case of infection, it is probable that the stabilizing effect on innate immune chemistry will be most effective if theophylline treatment is started towards the end of, or immediately after, antibiotic treatment so as to cause no blunting of the initial beneficial switch to a protective pro-inflammatory state as micro-organisms are destroyed, but before the detrimental prolonged tail of non-resolving inflammation is established. The ideal duration of treatment is not known but will probably be in the 3-6 weeks range, based on the observed duration of proinflammatory augmentation after episodes of exacerbation or infection. However, further light can only be shed on these practical issues by empirical research. It is the opinion of the authors that until conclusive evidence from clinical trials is available the use of low-dose theophylline to suppress inflammation can be justified in COPD, particularly during recovery from exacerbations and during pulmonary rehabilitation.

\section{Conclusion}

Theophylline and other MXs at low doses might be useful to reduce the potentially deleterious inflammatory burden during and after exacerbations of COPD, and in other pro-inflammatory states. There is potential to reduce the progression of muscle weakness, to delay or avoid overt sarcopenia, and to reduce the incidence, duration and intensity of low mood, delirium and illness behaviour, particularly in aged patients and those with multiple co-morbidities. The anti-sarcopenic properties of MXs could also be exploited for benefit as adjunctive treatment during pulmonary rehabilitation. Properly designed clinical trials are now needed to establish whether or not these effects are of consistent clinical utility.

Funding: none 
Conflicts of interest: none

Ethical approval: not applicable

\section{References}

1. Schultze-Weringhaus G, Meier-Sydow J. The clinical and pharmacological history of theophylline: first report on the bronchospasmolytic action in man by SR Hirsch in Frankfurt (Main) 1922. Clin Allergy 1982; 12: 211-5.

2. Barnes PJ. Theophylline. Pharmaceuticals (Basel) 2010; 3: 725-47.

3. Li JM. Fenton RA, Cutler BS, Dobson JG Jr. Adenosine enhances nitric oxide production by vascular endothelial cells. Am J Physiol 1995; 269: c519-23.

4. NICE clinical guideline CG101. Chronic obstructive pulmonary disease in the over 16s: diagnosis and management 2010. Available at www.nice.org.uk/guidance/CG101 (Accessed on: Feb 2, 2018)

5. NICE for British National Formulary. Theophylline. Available at https://bnf.nice.org.uk/drug/theophylline.html (Accessed on March 10, 2018)

6. Mascali JJ, Cvietusa P, Negri J, Borish L. Anti-inflammatory effects of theophylline: modulation of cytokine production. Ann Allergy Asthma Immunol 1996; 77: 34-8.

7. Allen SC. Systemic inflammation in the genesis of frailty and sarcopenia: An overview of the preventative and therapeutic role of exercise and the potential for drug treatments. Geriatrics 2017; 2(1): 6 .

8. Allen SC, Wong GTC, Vassallo M, Kwan JSK. Potential for a beneficial effect of theophylline on extended acute inflammation: review. Brit J Pharmaceut Res 2016; 14: 1-12.

9. Hoffman J, Ahira S. Innate immunity. Curr Opin Immunol 2013; 25: 1-3.

10. DeForge LE, Remick DG. Kinetics of TNF, IL-6 and IL-8 gene expression in LPS-stimulated human whole blood. Biochem Biophys Res Comm 1991; 174: 18-24.

11. Jansky L, Raymanova P, Kopecky J. Dynamics of cytokine production in human peripheral blood mononuclear cells stimulated by LPS or infected by Borrelia. Physiol Res 2003; 52: 593-8. 
12. Shaw AC, Joshi S, Greenwood H, Panda A. Aging of the innate immune system. Curr Opin Immunol 2010; 22: 507-13.

13. Yeh SS, Schuster MW. Geriatric cachexia: the role of cytokines. Am J Clin Nutr 1999; 70: 183-97. 14. Poon DCH, Ho YS, Chiu K, Chang CC. Cytokines: how important are they in mediating sickness? Neurosci Biobehav Rev 2013; 37: 1-10.

15. Vasunilashorn SM, Ngo L, Inouye SK, et al. Cytokines and postoperative delirium in older patients undergoing major elective surgery. J Gerontol A Biol Sci Med Sci 2015; 70: 1289-95.

16. Serhan CN, Brain SD, Buckley CD, et al. Resolution of inflammation: state of the art, definitions and terms. J Fed Amer Soc Exper Biol 2007; 21: 325-32.

17. Bruunsgaard H, Pedersen BK. Age-related inflammatory cytokines and disease. Immunol Allergy Clin North Am 2003; 23: 15-39.

18. Woods JA, Wilund KR, Martin SA, Kistler BM. Exercise, inflammation and aging. Aging Dis 2012; 3: 130-40.

19. Chung HY, Cesari M, Anton S, et al. Molecular inflammation: underpinnings of aging and agerelated diseases. Ageing Res Rev 2009; 8: 18-30.

20. Casperan CJ, Pereira MA, Curran KM. Changes in physical activity patterns in the United States, by sex and cross-sectional age. Med Sci Sports Exerc 2000; 32: 1601-9.

21. Cowie CC, Rust KF, Byrd-Holt DD, et al. Prevalence of diabetes and impaired fasting glucose in adults in the US population: NHANES survey 1999-2002.Diabetes Care 2006; 29: 1263-8.

22. Kalaria RN, Maestre GE, Arizaga R, et al. Alzheimer's disease and vascular dementia in developing countries: prevalence, management and risk factors. Lancet Neurol 2008; 7: 812-26.

23. Coresh J, Selvin E, Stevens LA, et al. Prevalence of chronic kidney disease in the United States. JAMA 2007; 298: 2038-47.

24. Dagenais S, Garbedian S, Wai EK. Systematic review of the prevalence of radiographic primary hip osteoarthritis. Clin Orthop Relat Res 2009; 467: 623-37.

25. Wouters EFM, Groenwegen KH, Dentener MA, Vernooy JHJ. Systemic inflammation in COPD: the role of exacerbation. Proc Am Thorac Soc 2007; 4: 626-34. 
26. Christian LM, Glaser R, Porter K, Malarkey WB, Beversdorf D, Kiecolt-Glaser JK. Poorer selfrelated health is associated with elevated inflammatory markers among older adults.

Psychoneuroendocrinology 2011; 36: 1495-504.

27. Michaud M, Balardy L, Moulis G, et al. Proinflammatory cytokines, aging and age-related diseases. J Amer Med Dir Assoc 2013; 14: 877-82.

28. Tousoulis D, Charakida M, Stefanadis C. Endothelial function and inflammation in coronary artery disease. Heart 2006; 91; 441-4.

29. Dantzer R, Cytokine sickness behaviour and depression. Immunol Allergy Clin North Am 2009; 29: 247-64.

30. Banks WA, Kastin AJ, Broadwell RD. Passage of cytokines across the blood-brain barrier. Neuroimmunomodulation 1995; 2: 241-8.

31. Banks WA. Blood-brain barrier transport of cytokines. Neuroimmune Biol 2008; 6: 93-107.

32. Yarlagadda A, Alfson E, Clayton AH. The blood-brain barrier and the role of cytokines in neuropsychiatry. Psychiatry (Edgmont) 2009; 6: 18-22.

33. Pan W, Stone KP, Hsuchou H, Manda VK, Kastin AJ. Cytokine signalling modulates blood-brain barrier function. Curr Pharm Des 2011; 17: 3729-40.

34. Tizard I. Sickness behaviour, its mechanisms and significance. Anim Health Res Rev 2008; 9: 8799.

35. Srinivasan D, Yen JH, Joseph DJ, Friedman W. Cell type-specific interleukin-1 beta signalling in the CNS. J Neurosci 2004; 24: 6482-8.

36. Carlson NG, Whitney WA, Chen J, Bacchi A, Rogers SW, Gahring LC. Inflammatory cytokines IL-1 alpha, IL-1 beta, IL-6 and TNF alpha impart neuroprotection to an excitotoxin through distinct pathways. J Immunol 1999; 163: 3963-8.

37. Arias $\mathrm{M}$, Khattab A, Allen S, Vassallo M, Kwan J. Inflammatory cytokines may mediate cognitive dysfunction and sickness behaviour during acute illness. J Neurolog Sci 2017; 381(suppl): 84.

38. Oikonomopoulou K, Ricklin D, Ward PA, Lambris JD. Interactions between coagulation and complement: their role in inflammation. Semin Immunopathol 2012; 34: 151-65.

39. Barnes PJ. Theophylline for COPD. Thorax 2006; 61: 742-4.

40. Barnes PJ. Theophylline in COPD. Proc Amer Thorac Soc 2005; 2: 334-9. 
41. Neuner P, Klosner G, Schauer E, et al. Pentoxyfylline in vivo down-regulates the release of IL-1 beta, IL-6, IL-8 and TNF alpha by human peripheral blood mononuclear cells. Immunology 1994; 83: 262-7.

42. Cvietusa P, Mascali JJ, Negri J, Borish L. Anti-inflammatory effects of theophylline: modulation of cytokine production. Ann Allergy Asthma Immunol 1996; 77: 34-8.

43. Ito K, Lim S, Caramori G, et al. A molecular mechanism of the action of theophylline: induction of histone deacetylase activity to decrease inflammatory gene expression. Proc Nat Acad Sci 2002; 99: 8921-6.

44. Ichiyami T, Hasegawa S, Matsubara T, Hayashi T, Furukawa S. Theophylline inhibits NF-kappa activation and I kappa B alpha degradation in human pulmonary epithelial cells. Arch Pharmacol 2001; 364: 558-61.

45. Subramanian V, Ragulan AB, Jindal A, Wiswhambar V. The study of tolerability and safety of theophylline given along with formoterol plus budesonide in COPD. J Clin Diagn Res 2015; 9: 10-13. 46. Markham A, Faulds D. Theophylline: A review of its potential steroid sparing effects in asthma. Drugs 1999; 56: 1081-91.

47. Barnes PJ. Theophylline: new perspectives for an old drug. Amer J Resp Crit Care Med 2003; 167: 813-8.

48. Pal M, Febbraio MA, Whitham M. From cytokine to myokine: the emerging role of IL-6 in metabolic regulation. Immunol Cell Biol 2014; 92: 331-39.

49. Pedersen BK, Febbraio M. Muscle-derived IL-6: a possible link between skeletal muscle, adipose tissue, liver and brain. Brain Behav Immun 2005; 19: 371-6.

50. Mikkelsen UR, Couppe C, Karlsen A, et al. Life-long endurance exercise in humans: markers and leg muscle size. Mech Ageing Dev 2013; 134: 531-40.

51. Woods JA, Veira VJ, Keylock KT. Exercise, inflammation and innate immunity. Immunol Allergy Rev 2006; 12: 6-33.

52. Pedersen BK. Exercise-induced myokines and their role in chronic disease. Brain Behav Immun 2011; 25: 811-6.

53. Brandt C, Pedersen BK. The role of exercise-induced myokines in muscle homeostasis and the defence against chronic diseases. J Biomed Biotechnol 2010; Article ID 520258: 6 pages. 
54. Spatafora M, Chiappara G, Merendino AM, D’Amico D, Bellia V, Bonsignore G. Theophylline supresses the release of TNF alpha by blood monocytes and alveolar macrophages. Eur Resp J 1994; 7: 223-8.

55. Yoshimura T, Usami E, Kurita C, et al. Effect of theophylline on the production of IL-1 beta, TNF alpha and IL-8 by human peripheral blood mononuclear cells. Biol Pharm Bull 1995; 18: 1405-8.

56. Bodera P, Stankiewicz W. Immunomodulatory properties of thalidomide analogs: pomalidomide and lenalidomide, experimental and therapeutic applications. Recent Pat Endocr Metab Immune Drug Discov 2011; 5: 192-6.

57. Eski M, Sahin I, Sengezer M, Serdar M, Ifran A. Thalidomide decreases the plasma levels of IL-1 and TNF following burn injury: is it the new drug for modulation of systemic inflammatory response. Burns 2008; 34: 104-8.

58. van der Borne BE, Dijkmans BA, de Rooij HH, le Cessie S, Verweij CL. Chloroquine and hydroxychloroquine equally affect TNF alpha, IL-6 and IF gamma production by peripheral blood mononuclear cells. J Rheumatol 1997; 24: 55-60.

59. Ohtsuka T, Hamada M, Hiasa G, et al. Effect of beta-blockers on circulating levels of inflammatory and anti-inflammatory cytokines in patients with dilated cardiomyopathy. J Am Coll Cardiol 2001; 37: 412-7.

60. Wu K, Tian S, Zhou H, Wu Y. Statins protect human endothelial cells from TNF-induced inflammation via ERK5 activation. Biochem Pharmacol 2013; 85: 1753-60.

61. Seymour HE, Worsley A, Smith JM, Thomas SHL. Anti-TNF agents for rheumatoid arthritis. Brit J Clin Pharmacol 2001; 51: 201-8.

62. Wedzicha JA, Calverley PMA, Rabe KF. Roflumilast: A review of its use in the treatment of COPD. Int J Chron Obstruct Pulmon Dis 2016; 11: 81-90.

63. Brooks P, Emery P, Evans JF, et al. Interpreting the clinical significance of the differential inhibition of COX-1 and COX-2. Rheumatology 1999; 38: 779-88.

64. Bailey JM. New mechanisms for the effects of anti-inflammatory glucocorticoids. Biofactors 1991; 3: 97-102.

65. Landi F, Marzetti E, Liperoti R, et al. NSAID use and sarcopenia in older people: the results of the iISIRENTE study. J Amer Med Dir Assoc 2013; 14: 626.e9-626.e13. 
Legend for figure 1. The immuno-modulatory effects of theophylline in sustained inflammation 